\title{
A UTILIZAÇÃO DE JOGOS DIDÁTICOS COMO AUXÍLIO REABILITATIVO A CRIANÇAS DEFICIENTES VISUAIS
}

\section{THE USE OF DIDACTIC GAMES AS A REHABILITATIVE AID FOR VISUALLY IMPAIRED CHILDREN}

\author{
João Cezar Silva Moura ${ }^{1}$, Bel. \\ Elton Moura Nickel², D.Sc. \\ Flávio Anthero Nunes Vianna dos Santos ${ }^{3}$, D.Sc. \\ (1) UDESC (Universidade do Estado de Santa Catarina) \\ e-mail: ioaocezarba@hotmail.com \\ (2) UDESC (Universidade do Estado de Santa Catarina) \\ e-mail: eltonnickel@gmail.com \\ (3) UDESC (Universidade do Estado de Santa Catarina) \\ e-mail:flavioanvs@hotmail.com
}

Palavras-chave: deficiência visual, jogos didáticos, reabilitação de crianças invisuais

Este artigo reúne estudos voltados à utilização de jogos enquanto recurso didático na reabilitação de crianças deficientes visuais e de seus aspectos físicos, cognitivos e sensoriais. São vistos conceitos que definem a deficiência visual, suas características e as dificuldades desta condição associadas ao universo infantil. Também são exploradas práticas de aprendizagem, bem como a classificação e atributos de jogos e didáticas utilizados nos processos reabilitativos. A coleta de dados se deu através de entrevistas semiestruturadas e observações sistemáticas em centros de reabilitação, tendo-se como objetivo a compreensão das atividades praticadas em conjunto com professores, orientadores e demais profissionais envolvidos. Por fim, as análises e conclusão foram realizadas mediante teorias e fundamentos de autores da área para identificar as principais contribuições que os jogos apresentam em todas as etapas deste processo.

Key-words: visual impairment, educational games, rehabilitation of blind children

This article brings together studies aimed at the use of games as a didactic resource in the rehabilitation of visually impaired children and their physical, cognitive and sensorial aspects. Concepts that define visual deficiency, its characteristics and the difficulties of this condition associated to the infant universe have also been studied. Learning practices, classifications and attributes of games and didactics used in rehabilitation processes were explored as well. Data was collected through semistructured interviews and systematic observations in rehabilitation centers, objectifying the understanding of the activities practiced together with teachers, counselors and other professionals. Finally, the analyzes and conclusion were carried out through theories and foundations of authors of the area to identify the main contributions that the games present in all stages of this process.

\section{Introdução}

Crianças com deficiências visuais (totais ou parciais, tanto congênitas quanto adquiridas) apresentam uma série de dificuldades durante seu desenvolvimento físico, cognitivo e sensorial, em especial devido à falta de materiais produzidos especificamente para o melhoramento destes 
atributos. Em tais situações, mostra-se efetiva a utilização de jogos didáticos como forma de auxílio reabilitativo à criança, pois apresentam características tanto lúdicas quanto educativas, ambas necessárias ao processo de reabilitação.

De acordo com relatórios emitidos pela Organização Mundial da Saúde (OMS, 2011), cerca de 285 milhões de pessoas em todo o mundo têm algum tipo de deficiência, 39 milhões apresentam cegueira (ou baixa visão) e $90 \%$ desta parcela da população vive em ambientes de baixa renda. Em âmbito nacional, 6,2\% dos brasileiros são considerados deficientes, dentre os quais 3,6\% têm algum tipo de deficiência visual (IBGE, 2013). Segundo dados disponibilizados pela Lions Club International Foundation (2016), cerca de 6 milhões de crianças em idade escolar têm algum tipo de deficiência visual e menos de uma a cada dez tem acesso à educação. Ainda segundo a mesma fonte, a taxa de desemprego deste grupo é entre 75 - 90\% (aproximadamente 5 vezes maior que a da população em geral).

Os obstáculos enfrentados por crianças deficientes visuais são diversos e podem vir a desmotivar e, até mesmo, impossibilitar sua permanência nas próprias instituições de ensino (OMS, 2011). Em meio a tais problemas (que incluem questões como transporte, infraestrutura, preparação profissional e adaptação de atividades diversas), observa-se a importância e, ao mesmo tempo, carência de didáticas específicas que integrem valores gerais de aprendizado e de desenvolvimento das habilidades consideradas essenciais para a adaptação do aluno à sua condição, conforme o Decreto 6.571 (Intervenções Estatais) e também segundo as exigências elaboradas e documentadas pela ABNT - NBR 9050 (referentes aos padrões das adaptações físicas para alunos com deficiência).

Diante deste contexto e dados apresentados, buscou-se, como objetivo geral, identificar as necessidades dos usuários relacionadas à problemática, ou seja, como auxiliar crianças deficientes visuais a se adaptarem à falta ou perda da visão (total e/ou parcial) através do desenvolvimento e treino de suas capacidades físicas, cognitivas e sensoriais remanescentes. Esta pesquisa também aponta as etapas pelas quais os estudos foram submetidos durante seu andamento, constando-se análises bibliográficas e de campo que possibilitaram a compreensão de conhecimentos teóricos e práticos, pretendendo-se, por fim, prover auxílio informacional aos profissionais, tutores e, inclusive, familiares envolvidos nos processos de reabilitação da criança.

\section{Referencial Teórico}

O referencial teórico foi composto por pesquisas em fontes diversas, abordando-se conceitos e definições através dos quais tornou-se possível compreender os principais temas abordados pela pesquisa. Para melhor compreensão das informações, estas foram subdivididas entre os seguintes tópicos:

- Deficiência - entendimento das principais características que distinguem a deficiência visual, como como suas influências no universo infantil;

- Teorias de Aprendizagem - princípios que explicam o modo como indivíduos assimilam o conhecimento e desenvolvem sua intelectualidade com o passar do tempo;

- Classificação dos Jogos - especificações utilizadas pelo mercado para classificação e distribuição dos jogos mediante diferentes faixas etárias.

\subsection{Deficiência}

De acordo com o Artigo 3 do Decreto $\mathrm{N}^{\circ} 3.298$, de 20 de Dezembro de 1999 - emanado pela Casa Civil (Presidência da República), tem-se por:

- Deficiência - toda perda ou anormalidade de uma estrutura ou função psicológica, fisiológica ou anatômica que gere incapacidade para o desempenho de atividade, dentro do padrão considerado normal para o ser humano;

- Deficiência permanente - aquela que ocorreu ou se estabilizou durante um período de tempo suficiente para não permitir recuperação ou ter probabilidade de que se altere, apesar de novos tratamentos;

- Incapacidade - uma redução efetiva e acentuada da capacidade de integração social, com necessidade de equipamentos, adaptações, meios 
ou recursos especiais para que a pessoa portadora de deficiência possa receber ou transmitir informações necessárias ao seu bem-estar pessoal e ao desempenho da função ou atividade a ser exercida (Código Civil, 1999).

\subsubsection{Deficiência Visual}

"Deficiência visual" é um termo abrangente e que se refere a toda e qualquer dificuldade voltada à capacidade que um determinado indivíduo tem com relação à visão.

De acordo com a Classificação Internacional de Deficiências, Incapacidades e Desvantagens (CIDID, 2004) a deficiência visual pode ser definida como: "[...] a perda total ou parcial, congênita ou adquirida, da visão". O nível de acuidade visual varia, determinando-se dois grupos de deficiência:

- Cegueira - há perda total da visão ou pouquíssima capacidade de enxergar, o que leva a pessoa a necessitar do Sistema Braille como meio de leitura e escrita.

- Baixa visão ou visão subnormal - caracteriza-se pelo comprometimento do funcionamento visual dos olhos, mesmo após tratamento ou correção. As pessoas com baixa visão podem ler textos impressos ampliados ou com uso de recursos óticos especiais.

De acordo com o Decreto $\mathrm{N}^{\circ} 5.296$ de 02 de Dezembro de 2004:

- Deficiência visual - cegueira na qual a acuidade visual é igual ou menor que 0,05 no melhor olho, com a melhor correção óptica; a baixa visão, que significa acuidade visual entre 0,3 e 0,05 no melhor olho, com a melhor correção óptica; os casos nos quais a somatória da medida do campo visual em ambos os olhos for igual ou menor que 60o; ou a ocorrência simultânea de quaisquer das condições anteriores.

\subsubsection{Deficiência Visual em Crianças}

A Organização Mundial da Saúde (OMS, 2011) relata que, anualmente, cerca de 500.000 crianças ficam cegas no mundo. Em geral, elas apresentam atraso global em seu desenvolvimento neuropsicomotor e visão funcional quando comparadas a crianças não acometidas por esta deficiência (SOUZA et al, 2010).

A cegueira em crianças é algo que vai além da mera falta de sua visão, contudo o auxílio dos pais, professores e cuidadores durante o estímulo de suas potencialidades pode fazer com que crianças invisuais levem uma vida normal, adquirindo características e habilidades inerentes ao desenvolvimento de qualquer pessoa. Para Santin e Simmons (1977), os conceitos, ações e fenômenos aos quais se deve estar atento neste sistema são:

Normalização - ênfase nas semelhanças (e não nas diferenças) e minimização dos efeitos da deficiência na educação (implementação do Braille);

- A Criança "Total" - compreensão das necessidades exclusivas durante o desenvolvimento de crianças deficientes visuais;

- O Conceito de Diferença - entendimento da deficiência visual como uma diferença, e não como uma insuficiência;

- Desenvolvimento Sensorial - compensação sensorial através da audição, tato e olfato;

- Primeira Infância - reconhecimento das limitações dos bebês durante os primeiros meses;

- Integração Visual - desenvolvimento das capacidades táteis da criança sem o auxílio associativo da visão;

- Auto-Percepção - discernimento do indivíduo com relação aos demais através de sua movimentação (elo intermediário para com o meio);

- Desenvolvimento Cognitivo e Afetivo construção de sua própria realidade (descrição e entendimento das características dos objetos);

- Solução de Problemas - incentivo à solução de problemas diversos por intermédio de estímulos cognitivos e sensoriais;

- Enfoque sobre a Audição - construção de um modelo de mundo com base nos sons; 
- Uso dos Pronomes Pessoais - superação da dificuldade ao referir-se a si mesmo e a terceiros através de pronomes (eu, você, ele/ela, nós, etc.);

- A Linguagem dos Pais - dependência de moderadores para construção de modelos mentais do mundo e interação com o meio;

- Ambiente Imprevisível - presença de fatores não controláveis e/ou perigosos no ambiente externo e que podem causar o retraimento da criança;

- Atividades Passivas - preferência por ambientes e atividades mais seguros que exigem pouca mobilidade e dependência de mediadores, respectivamente;

- Interação Social - complicações durante os processos de interação social com estranhos, amigos e, até mesmo, familiares;

- Má Interpretação - divergências entre a interpretação de mensagens durante a comunicação com pessoas visuais.

Crianças deficientes visuais processam e formulam informações de modo diferente. Por este motivo, torna-se fundamental a compreensão dos elementos que compõem suas singularidades perceptivas e comunicacionais.

\subsection{Teorias de Aprendizagem}

Dentre os vários princípios teóricos voltados ao ensino para crianças e suas diferentes abordagens, destaca-se a Epistemologia Genética de Jean Piaget, que se baseia na inteligência para elucidar como ocorrem os processos e etapas fundamentais durante a construção do conhecimento (PÁDUA, 2009).

Para Piaget, o desenvolvimento da inteligência humana pode ser dividido em quatro estágios distintos:

- Estágio sensório-motor (do nascimento aos dois anos) - a criança desenvolve um conjunto de "esquemas de ação" sobre o objeto que permitem a ela construir um conhecimento físico da realidade. É um período anterior à linguagem;

- Estágio pré-operatório (dos dois aos seis anos) - a criança inicia a construção da relação de causa e efeito, bem como suas simbolizações. É a chamada idade dos porquês e do faz-de-conta. Caracterizase pelo surgimento da capacidade de dominar a linguagem;

Estágio operatório-concreto (dos sete aos onze anos) - a criança começa a construir conceitos através de estruturas lógicas e constrói o conceito de número;

Estágio operatório-formal (dos onze aos dezesseis anos) - fase em que o adolescente constrói o pensamento abstrato e conceitual, conseguindo ter em conta as hipóteses possíveis, os diferentes pontos de vista e é capaz de pensar cientificamente.

\subsection{Classificação dos Jogos}

A predefinição de faixas etárias atribuídas aos vários tipos de jogos surge como uma maneira de facilitar a escolha dos mesmos para melhor atender às necessidades lúdicas e didáticas do usuário (crianças e adolescentes, no caso). De acordo com o Instituto Nacional de Metrologia, Normalização e Qualidade Industrial (INMETRO, 2011), os produtos destinados a crianças (linhas infantis) são recomendados de acordo com a idade.

- Bebês de colo e que engatinham - Desde o nascimento, os bebês são sensíveis ao seu meio ambiente e revelam que, ao nascer, suas percepções sensoriais respondem aos estímulos do olfato, paladar, som, tato e visão;

Bebês menores de 18 meses - Brinquedos vistosos e leves, de várias texturas, estimulam os sentidos da visão, da audição e do tato. Um móbile no berço diverte o bebê até que ele possa apanhar objetos;

- Crianças de 18 a 36 meses - Pequenas crianças irrequietas precisam de brinquedos que ativem seu movimento corporal, seja um carrinho grande para puxar, subir nele ou levar seus brinquedos dentro dele e qualquer objeto para subir ou cavalgar;

Pré-escolares de 3 a 6 anos - As crianças neste grupo etário são hábeis nos jogos de faz-de-conta, gostam de desempenhar papel de adulto e criar situações fantásticas; 
Crianças de 6 a 9 anos - Os jogos de tabuleiro, as bolinhas de gude e os brinquedos de armar colaboram no aprendizado das normas sociais;

- Crianças de 9 a 12 anos - Nesta idade, as crianças começam a desenvolver habilidades específicas e dirigem considerável atenção a certos passatempos e ocupações, modelos em escala, jogos de mágica, kits elaborados de peças de construção, de química, de experimentos científicos, enigmas e quebra-cabeças;

Adolescentes (após 12 anos) - após os 12 anos, os interesses dos jovens começam a mesclar-se com os dos adultos. Pode-se observar isto claramente no êxito crescente dos jogos eletrônicos e videogames mais complexos que, em geral, são considerados para toda a família.

Apesar de não serem levadas em conta as limitações específicas dos usuários deficientes visuais, ainda é possível identificar atributos que podem auxiliar os processos de reabilitação destinados aos mesmos, em especial no que diz respeito a questões físicas e cognitivas.

\section{Problemática}

\subsection{Reabilitação Através de Didáticas}

Para compreensão da extensão do problema, fêz-se necessária uma pesquisa voltada ao modo como os processos reabilitativos fazem uso de didáticas diversas. Segundo o guia "Understanding and Responding to Children's Needs in Inclusive Classrooms" (2009, p. 53), há uma série de estratégias que podem ser utilizadas pelo professor/instrutor com o objetivo de auxiliar a criança deficiente visual durante os processos de aprendizagem. São elas:

Braille - sistema de leitura composto por pontos em alto relevo numa superfície;

- Imagens táteis - desenhos em alto relevo sobre o papel braille;

- Ábaco / soroban - sistema para cálculos manuais aritméticos;

- Orientação de corpos e movimentação confiante - atividades físicas;

Utilização de bengala - requer treinamento e orientação de instrutores;

- Habilidades de vida diária - cozinhar, lavar roupas, higienização do corpo, etc.

Estas estratégias abordam diferentes aspectos relacionados ao desenvolvimento físico, cognitivo e sensorial de pessoas deficientes visuais. É possível, inclusive, perceber a importância dada aos exercícios que proporcionam o aumento da autonomia do indivíduo em seu cotidiano. Por este motivo, a utilização de jogos didáticos também apresenta um papel significativo nos processos de reabilitação.

\subsection{Centros de Reabilitação}

Os centros de reabilitação para deficientes visuais são instituições (organizações estruturadas) que prestam auxílio e atendimento às necessidades deste grupo singular. Entende-se por "reabilitação" os serviços que fornecem às pessoas com incapacidades a possibilidade de realizarem atividades de modo seguro, ativo e independente. De acordo com a Lions Club International Foundation (2016), alguns dos temas que contribuem com a melhora deste sistema são:

- Conscientização - informatização das implicâncias a respeito da deficiência visual aos profissionais, famílias e comunidade direta e indiretamente envolvidos na inclusão de pessoas cegas ou de baixa visão;

- Treinamento de Professores e Profissionais formação multidisciplinar e capacitação técnico procedimental aos especialistas comprometidas com a educação de pessoas deficientes visuais (escolas, centros de reabilitação, ambiente doméstico, etc.);

- Instalações, Equipamentos e Materiais infraestrutura adequada e recursos didáticos alternativos utilizados nos processos de habilitação e reabilitação. Muitas destas instituições são ONG's (Organizações Não Governamentais) sem 
fins lucrativos e, por este mesmo motivo, dependem da ajuda financeira de terceiros para continuarem a atuar. Já os centros pertencentes ou filiados ao governo dependem da disponibilização de verbas públicas (que nem sempre são suficientes para atender a todas as suas necessidades internas).

Devido a todos estes fatos e cenários, torna-se claro o porquê de muitos dos recursos utilizados nos processos de reabilitação para crianças deficientes visuais serem escassos, apresentarem condições precárias ou serem, ainda, de fabricação caseira (produtos adaptados), o que consequentemente pode influenciar sua eficácia e eficiência durante as atividades em que são utilizados.

\subsection{Definição do Problema}

Elucidar como ocorrem os processos de reabilitação de crianças deficientes visuais através da utilização de jogos didáticos nos centros reabilitativos para identificação e análise dos fatores que contribuem com o êxito deste processo.

\section{Metodologia}

As pesquisas foram feitas através de observações sistemáticas para reconhecimento dos padrões de comportamento das crianças durante a execução de tarefas diversas, atendo-se às suas dificuldades e desempenho geral. Também foram realizadas entrevistas semiestruturadas aos professores, especialistas e demais profissionais nos locais de estudo (TURATO, 2004). Por fim, foram realizadas análises dos dados (provenientes das pesquisas) para comparação e identificação dos principais aspectos relacionados à utilização de jogos didáticos como auxílio reabilitativo a crianças deficientes visuais.

\subsection{Locais de Estudo}

- Instituição $\mathrm{n}^{\circ} 01$

Localização: Balneário Camboriú (Santa Catarina)

- Instituição $n^{\circ} 02$

Localização: Itajaí (Santa Catarina)
- Motivo das escolhas: viabilidade no recolhimento de dados em âmbito didático/pedagógico

- Relações com o tema e problemática: presença de profissionais durante a realização de atividades que utilizam jogos didáticos para reabilitação de crianças deficientes visuais (totais e/ou parciais).

\subsection{Estudos dos Processos Reabilitativos}

O levantamento de dados utilizados para formular as entrevistas semiestruturadas e os checklists para observação das atividades nas instituições foi feito de acordo com os princípios teóricos abordados nas bibliografias e demais referências anteriormente citadas.

\section{Resultados das Pesquisas}

\subsection{Entrevistas Semiestruturadas}

A elaboração dos formulários para entrevista considerou os diversos problemas que acometem crianças deficientes visuais (anteriormente vistos na teorização e problematização da pesquisa). De acordo com as informações obtidas pelos profissionais entrevistados (4 pedagogos especializados em Educação Especial), a falta de recursos informacionais e de materiais específicos para abordagem reabilitativa da deficiência visual em crianças representa um dos maiores obstáculos a este processo. Isto faz com que muitos dos materiais utilizados sejam idealizados e/ou confeccionados pelos próprios colaboradores das instituições.

Relatou-se que, apesar dos conhecimentos acadêmicos e práticos contribuírem fortemente para a elaboração e manufatura destes materiais (que vão desde jogos didáticos até produtos adaptados para locomoção e manuseio de utensílios domésticos), estas ações não substituem a utilização de produtos no mercado projetados e fabricados exclusivamente para este fim.

Com relação aos métodos reabilitativos que utilizam jogos didáticos, estes são escolhidos de acordo com a capacidade física, cognitiva e sensorial de cada criança. Ainda de acordo com os instrutores, os principais aspectos trabalhados são: 
Cognição (percepção e reconhecimento das informações do meio);

Audição (percepção e reconhecimento dos sons externos);

Tato (percepção e reconhecimento das superfícies e texturas dos objetos);

- Sinestesia (combinação e associação de sensações);

Mobilidade (locomoção e transporte próprios);

- Interação social (relações com familiares e demais pessoas).

\subsection{Observações Sistemáticas}

As observações sistemáticas foram feitas com base nas informações obtidas através das entrevistas semiestruturadas. Após a elaboração de um checklist para conferência das atividades realizadas por 5 crianças (sob acompanhamento dos instrutores), verificou-se que os processos de reabilitação (nos quais os jogos didáticos estão inclusos) seguem, de modo sistemático, as seguintes etapas:

- $1^{\mathrm{a}}$ etapa - é feita uma avaliação preliminar considerando-se a idade da criança e, em seguida, são avaliadas suas capacidades físicas, cognitivas e sensoriais para escolha do jogo mais adequado;

- $2^{\mathrm{a}}$ etapa - apresentação do jogo à criança, onde ocorrem explicações das regras e objetivos a serem alcançados;

- $3^{\mathrm{a}}$ etapa - acompanhamento inicial do andamento do jogo para esclarecimento de possíveis dúvidas;

- $4^{\mathrm{a}}$ etapa - são feitas anotações referentes ao progresso e principais dificuldades da criança durante o jogo;

- $5^{\mathrm{a}}$ etapa - é feita uma pausa para relaxamento e conversação;

- $6^{\mathrm{a}}$ etapa - retoma-se o jogo até o momento em que este é finalizado ou até que os objetivos propostos sejam alcançados (total ou parcialmente);
- $7^{\mathrm{a}}$ etapa - é feita uma segunda avaliação referente ao progresso recente da criança.

\section{Análise de Dados}

Após a conclusão das pesquisas de campo (inquirições e observações), conclui-se que é possível estabelecer relações entre os elementos trabalhados na criança e as características funcionais que os jogos didáticos apresentam, tornando eficaz o processo de reabilitação com uso destes. Também é possível perceber que o método, ou seja, a ordem das etapas a serem seguidas neste processo, é de igual importância, pois considera a evolução da criança no decorrer do jogo e também em sessões variadas (diferentes dias na semana/mês), podendo-se mensurar mais precisamente os benefícios que a utilização de jogos didáticos proporcionam a curto, médio e longo prazo.

Segue abaixo uma listagem das relações entre os aspectos físicos, cognitivos e sensoriais da criança e os tipos de jogos didáticos recomendados, tendose como base os resultados obtidos nas observações e inquirições realizadas:

- Cognição - quebra-cabeças, jogos da memória (com diferentes formatos ou em alto/baixo relevo) e jogos-da-velha;

Audição - jogos com estímulos sonoros (chocalhos ou apitos, por exemplo) e atividades onde há emissão de sons que variam conforme a distância de sua emissão;

- Tato - jogos em que haja texturas de superfície variadas (altos/baixos relevos, panos com diferentes tipos de trama e maciez, tapetes táteis, tecidos e retalhos);

- Sinestesia - jogos que exijam orientação, mobilidade e posicionamento associados a sons, cheiros, noções de espaço e distâncias percorridas.

\section{Considerações Finais}

Ao final destes estudos, constatou-se que a utilização de jogos didáticos para reabilitação de crianças deficientes visuais é feita de acordo com fundamentações teóricas abordadas por muitos dos autores que tratam do assunto. Apesar das 
limitações mercadológicas e financeiras às quais este processo está submetidos na maioria das vezes, é possível afirmar que sua contribuição é indispensável para o aumento da autonomia e subsequente melhora na qualidade de vida de crianças deficientes visuais totais e/ou parciais.

\section{Sugestões para Estudos Futuros}

Os presentes estudos também pretendem prover embasamentos informacionais considerados necessários para a realização de pesquisas futuras (a serem conduzidas por demais pesquisadores interessados no assunto) nas quais a temática e suas ramificações possam ser ainda mais aprofundadas.

Segue abaixo uma listagem de sugestões para pesquisas futuras:

Análise de usabilidade das interações entre produto (jogos didáticos) e usuários (crianças deficientes visuais e instrutores);

Realização de estudos de caso para comparação de diferentes métodos utilizados na reabilitação de crianças deficientes visuais;

- Verificação das relações entre idade e desenvolvimento físico, cognitivo e sensorial de crianças invisuais durante a utilização de jogos didáticos.

- Conversão dos resultados obtidos em prérequisitos projetuais para elaboração e fabricação de jogos didáticos com fins reabilitativos.

\section{Referências Bibliográficas}

BIZ, V. A. et al. Processo de inclusão de alunos deficientes visuais na rede regular de ensino: confecção e utilização de recursos didáticos adaptados. Disponível em:

$<$ http://www.unesp.br/prograd/PDFNE2003/Proces so $\% 20 \mathrm{de} \% 20$ inclusao $\% 20 \mathrm{de} \% 20$ alunos\%20deficie ntes\%20visuais.pdf $>$. p. 6-7, 2002. Acesso em 23 out. 2016.

BRITO, P. R.; VIETZMAN, S. Causas de cegueira e baixa visão em crianças. Arq. Bras. Oftalmol., n.1, p. 49, 2000.
CAMPOS, L. M. L. C.; FELÍCIO, A. K. C.; BORTOLO, T. M. A produção de jogos didáticos para o ensino de ciências e biologia: uma proposta para favorecer a aprendizagem. Caderno dos Núcleos de Ensino, p. 48, 2003.

CUNHA, N. Brinquedo, desafio e descoberta. Rio de Janeiro: FAE, 1988.

GOLDENBERG, Mirian. A arte de pesquisar. Rio de Janeiro: Record, p.10, 2004.

GOMES, R. R.; FRIEDRICH, M. A Contribuição dos jogos didáticos na aprendizagem de conteúdos de Ciências e Biologia. Rio de Janeiro: Erebio, 2001.

INSTITUTO BRASILEIRO DE GEOGRAFIA E ESTATÍSTICA (IBGE). Disponível em: $<$ http://biblioteca.ibge.gov.br/visualizacao/livros/li v94522.pdf>. 2013. Acesso em 20 out. 2016.

INMETRO. Disponível em:

$<$ http://www.inmetro.gov.br/imprensa/releases/Inm etro-indica-brinquedos-mais-adequados-porfaixaetaria.pdf>. 2016. Acesso em 20 out. 2016

LAPLANE, A. L. F. de; BATISTA, C. G. Ver, não ver e aprender: a participação de crianças com baixa visão e cegueira na escola. Cad. CEDES [online], n.75, p. 11, 2008.

\section{LIONS CLUBS INTERNARTIONAL}

FOUNDATION. Disponível em:

$<$ http://www.lcif.org/PO/our-work/sight/educationand-rehabilitation.php $>$. 2016. Acesso em 20 out. 2016

MASINI, E. F. S. Educação do portador de deficiência visual: as perspectivas do vidente e do não vidente. Brasília: Ministério da Ação Social, CORDE, p. 62, 1993.

MIRANDA, S. No fascínio do jogo: a alegria de aprender. In: Ciência Hoje, p. 64. 2001.

ORGANIZAÇÃO MUNDIAL DA SAÚDE. The World Bank. Relatório Mundial Sobre a

Deficiência. Trad Lexicus Serviços Linguísticos. São Paulo: SEDPcD, p. 04-29, 2011.

PÁDUA, L. D. Epistemologia Genética de Jean 
Piaget. Revista FACEVV., n.2, p. 01, 2009.

PALÁCIO DO PLANALTO (Casa Civil), Decreto No 3.298, de 20 de dezembro de 1999. Disponível em: $<$ http://www.planalto.gov.br/ccivil_03/decreto/ d3298.htm>. 1999. Acesso em 20 out. 2016

PALÁCIO DO PLANALTO (Casa Civil), Decreto $\mathbf{N}^{0}$ 5.296, de 02 de dezembro de 2004. Disponível em: $<$ http://www.planalto.gov.br/ccivil_03/decreto/ d3298.htm>. 2004. Acesso em 20 out. 2016

PALÁCIO DO PLANALTO (Casa Civil), Decreto $N^{0}$ 6.571, de 17 de setembro de 2008. Disponível em:<http://www.planalto.gov.br/ccivil_03/_Ato200 7-2010/2008/Decreto/D6571.htm>. 2008. Acesso em 20 out. 2016

SANTIN, S.; SIMMONS, J. N. Problems in the construction of reality in congenitally blind children. Journal of Visual Impairment \& Blindness, Volume 71, 1977.

SOUZA et al. Descrição do desenvolvimento neuropsicomotor e visual de crianças com deficiência visual. Arq. Bras. Oftalmol., 2010.

TURATO, E. R. A questão da complementaridade e das diferenças entre métodos quantitativos e qualitativos de pesquisa: uma discussão epistemológica necessária. Em S. Grubits \& José A. V. Noriega (Orgs.), Método qualitativo: epistemologia, complementariedades e campos de aplicação. São Paulo: Vetor Editora, p. 17- 51, 2004.

UNESCO. Traduzido do inglês e digitado em São Paulo por Maria Amélia Vampré Xavier.

Understanding and responding to children's needs in inclusive classrooms. p. 53, 2009. World Health Organization International. Classification of impairments, disabilities, and handicaps: a manual of classification relating to the consequences of disease. Geneva, 2004.

\section{Agradecimentos}

Agradecimentos joviais à família, amigos, orientadores, professores, colegas, órgãos e instituição de ensino (CNPq, CAPES e UDESC) que, juntos, sempre farão parte desta eterna jornada pelo conhecimento. 